\title{
Staghorn calculus presenting as sterile pyuria
}

\author{
D Rowen, C A Carne, C Sonnex
}

\begin{abstract}
Urinary tract calculi are relatively common and usually symptomatic. We describe a patient who had several very unusual features with regard to his urinary tract calculus disease.
\end{abstract}

(Genitourin Med 1992;68:403-404)

\section{Introduction}

Staghorn calculi may present in a variety of ways. It is, however, unusual for patients with such calculi to present to genitourinary medicine (GUM) clinics. We describe a patient who on attending a GUM clinic was found to have sterile pyuria and on investigation was discovered to have a unilateral staghorn calculus.

\section{Case report}

A 33 year old, married, male heterosexual presented complaining of a recurrence of penile ulceration. He had contracted herpes simplex virus (HSV) infection affecting the penis seven years previously which had been confirmed on culture. He had subsequently experienced mild recurrences at approximately yearly intervals. The current attack had, however, lasted in excess of three weeks and had shown no signs of resolution.

He denied extramarital sexual intercourse or any homosexual activity. He had no history of sexually transmitted infection other than HSV, nor of urinary tract infection (UTI) $\mathrm{He}$ was employed as an airline steward on long haul flights. He had often visited Africa and the Far East. He has swum in lakes in Zimbabwe and South Africa but not in rivers or lakes of the Nile Delta. There was no family history of urinary tract disease; however, his grandfather was currently being treated for pulmonary tuberculosis.

On examination he was afebrile. There were several shallow serpiginous ulcers in the subpreputial region. There were no ulcers elsewhere. There was no significant regional lymphadenopathy. No urethral discharge was noted, and abdominal examination was normal.

Microscopy of a urethral smear showed 4-10 pus cells/hpf. A two-glass urine test yielded cloudy urine in both glasses which did not clear on the addition of $5 \%$ acetic acid. On standing there was a heavy sediment of cells. Microscopy of the urine revealed numerous neutrophils and a few red cells. No malignant cells were seen. On three separate occasions urines were negative on microscopy for bacteria including auramine phenol stained films for mycobacteria, and for schistosomes. Urine cultures, including for mycobacteria, were also negative on three occasions. Urethral cultures for $N$ gonorrhoeae, screening for chlamydia and herpes culture from the ulcers were also negative, as was syphilis serology. Microscopy of the stools demonstrated Endolimax nana cysts (non-pathogenic) but no ova or cysts of parasitic pathogens. Urea and electrolytes including calcium and phosphate were normal as was a full blood count. Abdominal radiographs revealed a staghorn calculus of the left kidney (fig 1). Isotopic renography demonstrated $22 \%$ of total renal function coming from the left kidney.

\section{Subsequent course}

Definitive surgical treatment has been delayed at the request of the patient. He remains asymptomatic with regard to his renal calculus. The presumed herpetic infection has resolved; however, urethral smears continued to show 4-10 pus cells/hpf and he was treated with oxytetracycline $500 \mathrm{mg}$ qds for two weeks. This has reduced but not totally eradicated the pus found on the urethral swab. His wife has also received antibiotic treatment. Repeated urine cultures have remained sterile but the pyuria persists. When travelling abroad he takes prophylactic antibiotics (trimethoprim $200 \mathrm{mg}$ bd). He has been advised to seek medical attention should he develop loin pain, dysuria, or become febrile.

\section{Discussion}

Sterile pyuria may be secondary to a number of infections, including syphilis and tuberculosis.

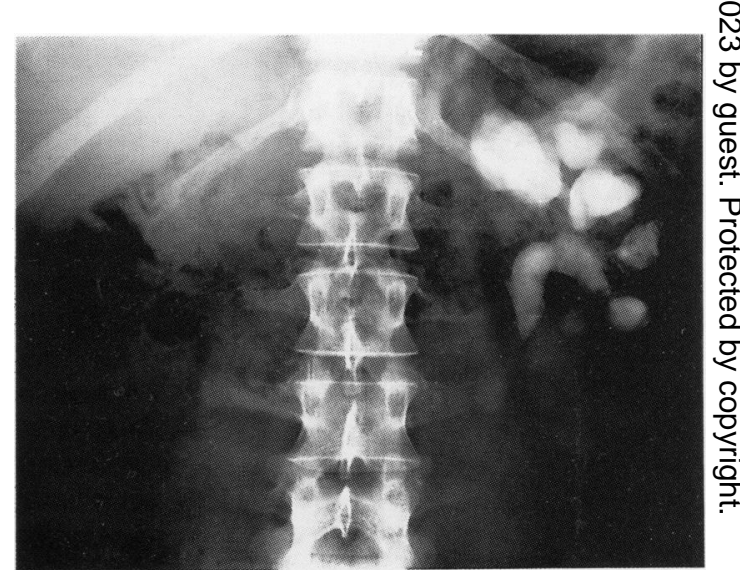

Figure Plain abdominal radiograph demonstrating staghorn calculus of left kidney.

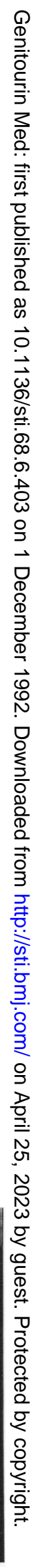


Syphilis has been effectively excluded in this case on the basis of repeated negative serology. Renal tract tuberculosis clearly needed to be excluded, especially in view of the family history of pulmonary tuberculosis. Culture for the organism in early morning samples of urine is mandatory as negative auramine phenol stained films of urinary sediment for mycobacteria do not adequately exclude infection.

Urinary tract calculi are relatively common in the western world and their incidence as estimated from hospital admission data appears to be increasing. ${ }^{1}$ Renal stones occur more commonly in patients from the developed world ${ }^{2}$ whereas bladder stones are more likely to be found in patients from the developing world. The composition of calculi is variable; in the UK the most abundant and frequently occurring constituents are calcium oxalate mono and dihydrate and their apatites, and calcium phosphates. ${ }^{2}$

Numerous factors have been postulated as being of aetiological importance in the development of urolithiasis. These include preexisting structural or metabolic abnormalities, infection, occupation and recreational activities. ${ }^{3-7}$ History and investigations do not suggest that our patient has abnormalities of structure or metabolism which may have predisposed him to develop renal calculi, nor is there a history of episodes of infection which could have been an initiating factor or to have occurred as a consequence of the stone. One may postulate that as an airline steward, our patient could be in danger of intermittent, mild dehydration due to frequent stop overs in the tropics and sub-tropics; however, even prior to the diagnosis being made he was aware of this possibility and accordingly increased his fluid intake.

Urinary tract infection may, albeit rarely, be accompanied by urethritis and urethral discharge. What is not immediately clear in this case if whether the pus found on urethral swabbing was secondary to his presumed herpetic infection or due to a bacterial urethritis or as a consequence of his marked pyuria. Urethral swabs have however consistently shown pus cells despite resolution of the penile ulceration, and his treatment with antibiotics.
These findings support the hypothesis that the urethral pus is a result of the gross pyuria.

Staghorn calculi are rarely silent. Singh $e t a l^{8}$ estimated from post mortem findings the incidence of silent staghorn calculi to be $0.05 \%$. Spontaneous disappearance of such calculi in the absence of long term antimicrobial chemotherapy has been described. ${ }^{9}$ The patient described had no symptoms which were attributable to the calculus. He was not aware of his urine being cloudy. This calculus was, and has remained silent in as much as the patient is asymptomatic. Despite this surgical intervention is planned for the near future as calculi predispose to infection which could result in further loss of function in the affected kidney or more seriously a septicaemic episode. Furthermore, once infection in the presence of lithiasis becomes established, attempts to sterilise the urine with antibiotics often fail. $^{610}$

This case is unusual with respect to the large volume of pus excreted in the urine in the absence of infection, and that a calculus of this size has remained asymptomatic. This emphasises the importance of a visual inspection of a urine sample in a GUM clinic and of the need to perform a full investigation in all cases of sterile pyuria, which should include plain abdominal radiographs or ultrasound scans even when the patient is asymptomatic.

1 Department of Health. Hospital Episode System. Returns for $1987 / 8,1988 / 9,1989 / 90$

2 Sutor DJ, Wooley SE, Illingworth JS. A geographical and historical survey of the composition of urinary stones. $B$ f Urol 1974;45:393-407.

3 Maynard JF. Case profile. Nephrocalcinosis with ureterocalcinosis due to medullary sponge kidney. Urology 1980;15:310-1.

4 Currie WJG, Turmer P. The Frequency of renal stones in Great Britain in a gouty and non-gouty population. $\mathrm{Br} \mathcal{F}$ Urol 1979;51:337-41.

5 Resnick M, Durward BA, Pridgen BS, Goodman HD. Genetic predisposition to formation of calcium oxalate Genetic predisposition to formation of calcium

6 Lerner SP, Gleeson MJ, Griffith DP. Infection stones. 7 Urol 1989;141:753-8.

7 Rose GA. Medical Investigations and Screening Procedures for Urinary Stones. In: Chisholm GD, Fair WR, eds. Scientific Basis of Urology. Heinemann Medical Books, 1990.

8 Singh M, Chapman R, Tresidder GC, Blandy J. The fate of unoperated staghorn calculus. Br f Urol 1973;45:581-5.

9 McGann G, Grant D, Miller R. Spontaneous disappearance of staghorn calculi. Br $\mathcal{F}$ Radiol 1991;64:1161-2.

10 Griffith DP, Moskowitz PA Carlton CE Adjunctive chemotherapy of infection-induced staghorn calculi. $f$ Urol 1979;121:711-5. 